\title{
Increasing Discrimination of DEA Evaluation by Utilizing Distances
}

\author{
to Anti-efficient DEA Frontiers \\ Wan-fang Shen ${ }^{\mathrm{a}}$, Da-qun Zhang ${ }^{\mathrm{b}}$, Wen-bin Liu ${ }^{\mathrm{c}}$, Guo-liang Yang ${ }^{\mathrm{d} *}$ \\ ${ }^{a}$ School of Mathematic and Quantitative Economics, Shandong University of Finance and \\ Economics, Jinan 240014,China \\ ${ }^{\mathrm{b}}$ Fox School of Business and Management, Temple University, Philadelphia 19122, USA \\ ${ }^{c}$ Kent Business School, University of Kent, Canterbury CT2 7PE, UK \\ ${ }^{\mathrm{d}}$ Institute of Policy and Management, Chinese Academy of Sciences, Beijing 100190, China
}

\begin{abstract}
This paper develops three DEA performance indicators for the purpose of performance ranking by using the distances to both the efficient frontier and the antiefficient frontier to enhance discrimination power of DEA analysis. The standard DEA models and the Inverted DEA models are used to identify the efficient and anti-efficient frontiers respectively. Important issues like possible intersections of the two frontiers are discussed. Empirical studies show that these indicators indeed have much more discrimination power than that of standard DEA models, and produce consistent ranks. Furthermore, three types of simulation experiments under general conditions are carried out in order to test the performance and characterization of the indicators. The simulation results show that the averages of both the Pearson and Spearman correlation coefficients between true efficiency and indicators are higher than those of true efficiency and efficiency scores estimated by the BCC model when sample size is small.
\end{abstract} Keyword: Data envelopment analysis; Discrimination; Efficient Frontier; Antiefficient Frontier.

${ }^{*}$ Corresponding author. Address: Institute of Policy and Management, Chinese Academy of Sciences, P.O.Box 8712, Beijing, 100190, China. Tel.: +86-10-59358423; E-mail addresses: glyang@ casipm.ac.cn (G.L.Yang). 


\section{Introduction}

Data envelopment analysis (DEA) was first introduced by Charnes et al. (1978), and has been widely used in performance or productivity evaluation. The main idea of the classic DEA is to first identify the production frontier on which the decision making units (DMUs) will be regarded as efficient. Then those DMUs not on the frontier will be compared with their peers on the frontier to estimate their efficiency scores. All the DMUs on the frontier are deemed to have the same level of performance and to represent the best practice. One of the main advantages of DEA is to allow the DMUs to have full freedom to select their weights, which are most favorable for their assessments to achieve the maximum efficiency score. This full flexibility of selecting weights is important in the identification of inefficient DMUs. However, this full flexibility may much reduce the discrimination power of DEA in the sense that there often exist too many DMUs on the frontier, which cannot be further ranked in the standard DEA models. When there are many input and output variables but only a few DMUs are available, decision makers (DMs) may find that all or most DMUs are efficient, and such results would be of little use for decision making. As Alder et al (2002, p.250) argued, "Often decision-makers are interested in a complete ranking, beyond the dichotomized classification, in order to refine the evaluation of the units."

Regarding the number of DMUs required in DEA models, Cooper et al. (2000, p. 252) proposed a rule of thumb, which demands

$$
n \geq \max \{m \times s, 3(m+s)\},
$$

where $n$ is the number of DMUs, $m$ and $s$ are the number of inputs and outputs. However, the rule above is sometimes violated in reality, because of small DMUs sample but many input and output variables. In such case, the standard DEA models are not as useful as expected.

Therefore, many researchers have sought to improve the discrimination capability of standard DEA models. Now there are three main areas in DEA literature: The first area requires preferential or prior information from relevant decision-makers to enhance the discrimination ability of DEA models. For example, some scholars have 
developed the weights restriction (Allen et al. 1997; Thanassoulis et al. 2004) or preference change methods (Liu et al. 2006; Zhang et al. 2009; Meng et al. 2008) to incorporate the prior information or value judgments of DMs into DEA models. The second area is based on cross-efficiency matrix, in which DMUs are evaluated by both itself and other peers (Sexton et al. 1986; Doyle and Green 1994). Although crossefficiency method is often very useful, in our opinion, the cross-efficiency scores have moved quite away from the basic principle of DEA. For instance in the case of one standard input variable, all the DMUs in fact use the same weights to compute their cross-efficiency scores. The third popular area is the super-efficiency method, which computes the score of the DMU being evaluated by excluding itself from the reference set (Banker and Gifford 1988; Andersen and Petersen 1993). It is clear that this model uses different reference sets to evaluate the efficient DMUs and inefficient DMUs. Furthermore, Banker and Chang (2006) reported that Andersen and Petersen's (1993) procedure using the super-efficiency scores for ranking efficient observations had poor performance.

Whilst each technique is useful in a specialist area, no one can be referred to as a complete solution to all problems. In this paper we explore another idea to enhance the discrimination power of DEA. People often have more than one reference point of view in judging DMUs. That is they do not just compare the DMUs with good references, but sometimes with bad references as well. In other words, on one hand a DMU is better if it is closer to the good references (or efficient frontier); on the other hand, it is also good if it is far from the bad references (or anti-efficient frontier). In this sense, the standard DEA models have just employed the best practice DMUs to construct the efficient frontier and haven't fully taken the advantage of the information implied in the data. The earliest work on anti-efficient frontier can be traced to "Inverted" DEA model proposed by Yamada et al. (1994). Compared to the standard DEA models which evaluate DMUs from the perspective of optimism, "Inverted" DEA model is to evaluate the performance of DMUs from the perspective of pessimism. Recently, some scholars employed Inverted DEA model to exploit more information from the data in their applications. For example, Takamura and Tone (2003) employed the DEA and Inverted 
DEA with weights restriction to solve the problem of site selection for the relocation of several government agencies outside of Tokyo. Paradi et al. (2004) used DEA and Inverted DEA models, which are so called "Worst Practice DEA" in the paper, to identify the worst practices in banking credit analysis. By using some layering or peeling technique (Thanassoulis 1999), the proposed approach increased the classification accuracies through the elimination of self-identifiers. Johnson and McGinnis (2008) employed both the efficient and anti-efficient frontiers to identify outliers and thus improve the accuracy of estimators in the second stage regression analysis.

In addition, some scholars tried to construct some new efficiency measures based on DEA and Inverted DEA models. Entani et al. (2002) employed both DEA and Inverted DEA models to obtain the upper and lower bound of interval efficiency of DMUs. They argued if the range of the interval efficiency is large, then it means that although the DMU performs good from the optimistic viewpoint, it performs bad from the perspective of pessimistic. Then they used the interval efficiency to obtain a partialorder relation of DMUs. Wang and Luo (2006) and Wu (2006) constructed the best and worst virtual DMUs as TOPSIS does (Hwang and Yoon 1981), and simply add them into the existing DMU set to carry out further DEA and Inverted DEA analysis using the extended data set. However, it may not be a wise idea because the Production Possibility Set (PPS) will be greatly changed in this case. Amirteimoori (2007) ${ }^{1}$ employed the Inverted DEA models to define the anti-efficient frontier. Then he used slacks based DEA and Inverted models to measure the weighted $\mathrm{L}_{1}$-distances from $\mathrm{DMU}_{0}$ to both efficient and anti-efficient frontier. Finally, he defined a new combined efficiency measure based on the two distances to rank DMUs. However, since the efficiency scores of these DMUs on efficient frontier and anti-efficient frontier are 1 and -1 respectively, this combined efficiency measure is not able to improve discrimination power of DEA models either. Furthermore, there is no justification that the combined efficiency measure performs better than existing ones. Zhou et al. (2007)

\footnotetext{
1 Note: In our view, there are some typos (errors) on inequalities in model (8) and model (10) in Amirteimoori (2007).
} 
used the DEA model without explicit inputs (see, e.g., Meng et al. 2005, Liu et al. 2011) to combine the efficient and anti-efficient measures to rank the DMUs. However we can easily verify that their approach cannot increase significantly the discrimination power of DEA models.

In this paper, we develop another DEA approach based on the idea of utilizing both good and bad frontiers to enhance discrimination power of DEA. The remainder of the paper is organized as follows: in Section 2, we discuss the approaches that can identify the anti-efficient frontier of DMUs. Furthermore, in this section, we introduce three composite performance indicators to combine the information from both best and worst viewpoints; In Section 3, we provide two empirical studies to illustrate the features of the indicators, and then we carry out simulation studies to examine the performance of our composite indicators in Section 4. Finally, conclusions and discussions are given in Section 5.

\section{Ranking DMUs via both Efficient and Anti-efficient Frontiers}

In this section we first outline our approach. For simplicity, we will illustrate the idea based on the radial measurement. Let $X=\left(x_{1}, x_{2}, \ldots, x_{m}\right)$ and $Y=\left(y_{1}, y_{2}, \ldots, y_{s}\right)$ be input and output vectors of $m$ and $s$ dimension respectively. Then Production Possibility Set (PPS) is defined by

$$
P P S=\{(X, Y): X \text { can produce } Y\} .
$$

The boundary of PPS is referred to as production technology or production frontier. Note, this unobservable production frontier is called true frontier or true efficient frontier hereinafter. When output is single, the production frontier is called production function in economic literature. DMUs which are technically efficient operate along the frontier, while those technically inefficient DMUs operate at points in the interior of $P P S$. Thus it is rational to rank DMUs according to their distances to the true frontier.

Let $\left\{\left(x_{j}, y_{j}\right) \mid j=1, \ldots, n\right\}$ be a group of observed input and output data. Based on such observations, DEA models construct a piecewise linear production frontier, a nonparametric estimate of the unobservable true frontier. Then DEA models measure the 
efficiency of a DMU via its distance to the estimated frontier. Here we restate the inputoriented CCR model with slacks of inputs and outputs as follows.

$$
\begin{aligned}
& h_{b}^{*}=\min \quad \theta-\varepsilon\left(\sum_{i=1}^{m} s_{i}^{-}+\sum_{r=1}^{s} s_{r}^{+}\right) \\
& \text {s.t. }\left\{\begin{array}{c}
\sum_{j=1}^{n} x_{i j} \lambda_{j}+s_{i}^{-}=\theta x_{i 0}, i=1, \ldots, m, \\
\sum_{j=1}^{n} y_{r j} \lambda_{j}-s_{r}^{+}=y_{r 0}, r=1, \ldots, s, \\
\lambda_{j}, s_{i}^{-}, s_{r}^{+} \geq 0, j=1, \ldots, n .
\end{array}\right.
\end{aligned}
$$

where $\varepsilon$ is a non-Archimedean infinitesimal.

In theory, Banker (1993) provided a formal statistical foundation for DEA and argued that while the efficient frontier is biased below the true efficient frontier for a finite sample size, the bias goes zero for large samples. However when sample size is small, the estimated frontier could be far away from the true one so that the efficiency scores of DMUs are much higher than their true efficiency scores. For instance, many DMUs are on the estimated frontier and cannot be discriminated although some of them are in fact quite far from the true frontier.

\subsection{The Main Ideas}

To solve such a problem, one of the possibilities is to utilize information of antiefficient frontier. Anti-efficient frontier is the worst practice frontier constructed by worst practice DMUs. To identify anti-efficient frontier, we here simply treat the inputs and outputs of DMUs both as undesirable variables, and then use some DEA models with undesirable inputs and outputs. The idea is simple: if the inputs and outputs of DMUs are undesirable, one should maximize the inputs and minimize the outputs to find the efficient frontier. Using radial measurement and input orientation, this idea leads to the following Inverted DEA (CCR type) model: 


$$
\begin{aligned}
& h_{w}^{*}=\mathrm{M} \text { a x } \theta \\
& \text { s.t. }\left\{\begin{array}{l}
\sum_{j=1}^{n} x_{i} \lambda \geq \theta x_{0}, \dot{i} 1, \ldots m \\
\sum_{j=1}^{n} y_{j} \leq y_{r},=r \quad 1, \ldots s \\
\lambda_{j} \geq 0, j=1, n \ldots, .
\end{array}\right.
\end{aligned}
$$

Here $h_{w}^{*}$ measure the degree of inefficiency or anti-efficiency by radial measurement. Similarly, we can also have the following output oriented DEA model:

$$
\begin{gathered}
\left(h_{w}^{*}\right)^{-1}=\operatorname{Min} \quad \phi \\
\text { s.t. }\left\{\begin{array}{c}
\sum_{j=1}^{n} x_{i j} \lambda_{j} \geq x_{i 0}, i=1, \ldots, m, \\
\sum_{j=1}^{n} y_{r j} \lambda_{j} \leq \phi y_{r 0}, r=1, \ldots, s, \\
\lambda_{j} \geq 0, j=1, \ldots, n .
\end{array}\right.
\end{gathered}
$$

If we wish to consider the slacks of inputs and outputs, we can then introduce some variables $s_{i}^{+}, s_{r}^{-}$, and transform model (2) to the following model:

$$
\begin{aligned}
& h_{w}^{*}=\operatorname{Max} \quad \theta-\varepsilon\left(\sum_{i=1}^{m} s_{i}^{-}+\sum_{r=1}^{s} s_{r}^{+}\right) \\
& \text {s.t. }\left\{\begin{array}{c}
\sum_{j=1}^{n} x_{i j} \lambda_{j}-s_{i}^{-}=\theta x_{i 0}, i=1, \ldots, m, \\
\sum_{j=1}^{n} y_{r j} \lambda_{j}+s_{r}^{+}=y_{r 0}, r=1, \ldots, s, \\
\lambda_{j}, s_{i}^{-}, s_{r}^{+} \geq 0, j=1, \ldots, n .
\end{array}\right.
\end{aligned}
$$

Let us emphasize that there are many different approaches to deal with undesirable variables in DEA (see e.g., Liu et al 2010 for a summary). However here we only explore this simpler approach.

Based on the Inverted DEA models above, we can obtain the Anti-Production Possibility Set (APPS) as follows: 


$$
A P P S=\left\{(x, y) \mid \sum_{j=1}^{n} x_{j} \lambda_{j} \geq x, \sum_{j=1}^{n} y_{j} \lambda_{j} \leq y, \lambda_{j} \geq 0\right\}
$$

Definition 1: Anti-efficient frontier is the boundary of APPS.

Obviously, if $h_{w}^{*}=1$ in model (1), then the DMU is on the anti-efficient frontier. Thus we introduce another two definitions. Note, $h_{w}^{*}$ is called anti-efficiency score in following sections.

Definition 2: $\mathrm{DMU} \mathrm{U}_{0}$ is weakly anti-efficient, if and only if $h_{w}^{*}=1$ in model (2).

Definition3: $\mathrm{DMU}_{0}$ is strongly anti-efficient, if and only if $h_{w}^{*}=1$, and $s_{i}^{+^{*}}=s_{r}^{-^{*}}=0$ in model (4).

Theorem 1: The optimal value of model (3) is the reciprocal of that of model (2).

Proof: If we let $\lambda_{j}^{\prime}=\lambda_{j} / \theta$, then we can rewrite model (2) as follows:

$$
\begin{aligned}
& \mathrm{M} \text { a } \\
& \text { s.t. } \theta \\
& \sum_{j=1}^{n} x_{i} \lambda^{\prime} \geq x_{0}, \dot{i} 1, \ldots m \\
& \sum_{j=1}^{n} y_{j}^{\prime} \leq \frac{1}{\theta} \quad y_{r},=r \quad 1, . \\
& \lambda_{j}^{\prime} \geq 0, j=1, n \ldots,
\end{aligned}
$$

By changing the object function $\theta$ to $1 / \theta$, the model (5) can be transformed to:

$$
\begin{gathered}
\text { Min } \frac{1}{\theta} \\
\text { s.t. }\left\{\begin{array}{c}
\sum_{j=1}^{n} x_{i j} \lambda_{j}^{\prime} \geq x_{i 0}, i=1, \ldots, m, \\
\sum_{j=1}^{n} y_{r j} \lambda_{j}^{\prime} \leq \frac{1}{\theta} y_{r 0}, r=1, \ldots, s, \\
\lambda_{j}^{\prime} \geq 0, j=1, \ldots, n .
\end{array}\right.
\end{gathered}
$$

Hence if $\theta^{*}$ is the optimal solution of model (6), it is also the optimal solution of model (5). And then it is also the optimal solution of model (2). Hence the optimal value of Model (6) is the reciprocal of that of model (2). 
If we let $\phi=1 / \theta$, then model (6) can be written as model (3) that is the optimal value of model (3) is also the reciprocal of that of model (2).

Let us note that the dual model of model (2) reads:

$$
\begin{gathered}
\operatorname{Min} \begin{array}{c}
u^{t} y_{0} / v^{t} x_{0} \\
\text { s.t. }\left\{\begin{array}{c}
u^{t} y_{j} / v^{t} x_{j} \geq 1, \\
u \geq 0, v \geq 0, j=1, \ldots, n .
\end{array}\right.
\end{array}
\end{gathered}
$$

where $x_{j}, y_{j}, v, u$ are the inputs, outputs of $\mathrm{DMU}_{j}$ and the corresponding weights respectively. Due to minimizing the ratio score, the Inverted DEA model (7) can be viewed to evaluate the performance of DMUs from the perspective of pessimism.

To illustrate the geometric meanings of the efficient and anti-efficient frontiers, we assume there are only two inputs and one output, and all of them are desirable. As Figure 1 shows, contrary to the DEA models using the best practice DMUs A, B, C and F to construct the efficient frontier, Inverted DEA model (2) employ the worst practice DMUs A, D, E and F to construct the anti-efficient frontier. Therefore, we can let the DMU being evaluated compare with virtual or real DMUs located on both efficient and anti-efficient frontiers. For instance, we can identify the peers G' and G' of DMU G, which are separately located on efficient and anti-efficient frontiers. Then we can measure the distances from G to G' (or G') by using radial measurement OG'/OG (or OG'/OG). The larger OG'/OG, DMU G being evaluated is nearer to efficient frontier, and thus performs better. Meanwhile, the larger OG"/OG, DMU G is farther from the anti-efficient frontier, and thus performs better. As for these efficient DMUs A, B, C and F, A and F can be regarded worse than the other efficient DMUs on the efficient frontier as $\mathrm{A}$ and $\mathrm{F}$ are also on the anti-efficient frontier. 


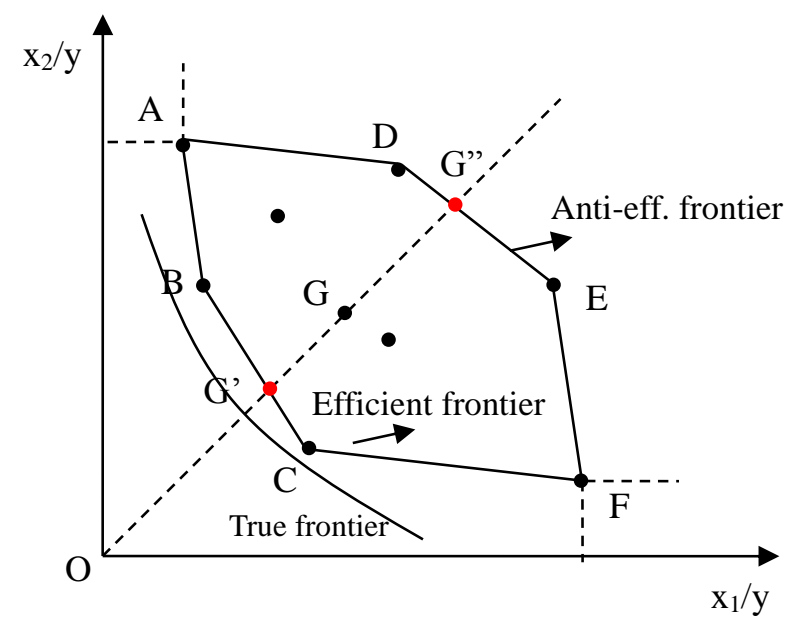

Figure 1: Distances to Efficient and Anti-Efficient Frontiers

Therefore, by utilizing the anti-efficient frontier generated by the worst practice DMUs, we can obtain more information about performance of DMUs, and thus enhance power of discrimination for DEA analysis. In the following section, we will present three intuitive approaches based on the radial measurement to utilize this extra information in DEA analysis.

\subsection{DEA Performance Indicators}

There are various possible ways to utilize the information of both the efficient and anti-efficient frontiers. However, to examine such idea, here we only propose three intuitive approaches to aggregate the radial based efficiency score $h_{b}^{*}, h_{w}^{*}$ of the inputoriented CCR (BCC) and Inverted CCR DEA models. It is possible to adopt the idea used in TOPSIS method to construct indicators of the form: $d b /(d g+d b)$, where $d g$ and $\mathrm{db}$ are the distances to the good and bad frontiers respectively. However it is clear that these indicators will take the same values on the two frontiers so that it is impossible to produce a full rank for their performance. To this end we have to use different ideas.

In the first approach, we define a composite DEA indicator as $h_{I}^{*}=h_{b}^{*}+h_{w}^{*} \cdot \varepsilon$, where $\varepsilon$ is the non-Archimedean infinitesimal. Hereinafter, $h_{I}^{*}$ is called "CDI-I". Naturally, people may argue it is more important to be close to the best frontier, and less important to be far from the worst frontier. Hence if people think the efficiency score $h_{b}^{*}$ is overwhelmingly more important than anti-efficiency score $h_{w}^{*}$, then we can 
use $h_{I}^{*}=h_{b}^{*}+h_{w}^{*} \cdot \varepsilon$ to rank the DMUs with the Lexicographic order. For example, if we wish to evaluate $\mathrm{DMU}_{1}$ and $\mathrm{DMU}_{2}$, we first use $h_{b}^{*}$ to compare their performance. If $h_{1 b}^{*}>h_{2 b}^{*}$, then DMU 1 is considered to perform better than $\mathrm{DMU}_{2}$. Otherwise if $h_{1 b}^{*}=h_{2 b}^{*}$, then we use $h_{w}^{*}$ to compare $\mathrm{DMU}_{1}$ and $\mathrm{DMU}_{2}$. If $h_{1 w}^{*}>h_{2 w}^{*}$, then $\mathrm{DMU}_{1}$ performs better than $\mathrm{DMU}_{2}$. Note that this index is almost the same as the standard DEA score except for those efficient DMUs.

In the second approach, we treat the two scores more equally and define another composite DEA indicator by combining the two as $h_{I I}^{*}=\left[h_{b}^{*}+\left(1-1 / h_{w}^{*}\right)\right] / 2$, so called “CDI-II".

Let us note that it may not be a good idea to directly use $h_{b}^{*}+h_{w}^{*}$ because the ranges of $h_{b}^{*}$ and $h_{w}^{*}$ are different. The range of $h_{b}^{*}$ is $(0,1]$ and that of $h_{w}^{*}$ is $[1,+\infty)$. We wish the latter term to have the same orientation as $h_{b}^{*}$ (i.e. the larger is better). Therefore, we need to use some transformation before we can add the two scores. The range of the score $h_{w}^{*}$ is normally $[1,+\infty)$, and $1 / h_{w}^{*}$ is the optimal value of the output-oriented anti-efficient CCR DEA models according to Theorem 1. Thus it is reasonable to apply transformation $1-1 / h_{w}^{*}$ as we have used in this paper. In this case if $\mathrm{DMU}_{0}$ is on the anti-efficient frontier, then $h_{w}^{*}=1$ and $h_{I I}^{*}=h_{b}^{*} / 2 \leq 1 / 2$. If a DMU is on both the efficient and anti-efficient frontiers like A and F in Fig.1, we have $h_{b}^{*}=1$, $h_{w}^{*}=1$ and then $h_{I I}^{*}=1 / 2^{2}$. And if a DMU is on the efficient frontier but not on the anti-efficient frontier, then its score will be higher than $1 / 2$, and thus it performs better than these DMUs located on both the efficient and anti-efficient frontiers.

As for CDI-I, $h_{b}^{*}$ has the dominate importance with respect to $h_{w}^{*}$. For CDI-II, we can show that $h_{b}^{*}$ still plays a more important role than $h_{w}^{*}$ by examining the partial derivatives of the CDI-II as follows:

\footnotetext{
${ }^{2}$ It should be noted that if there is a need to further rank the DMUs on both efficient and anti-efficient frontiers, we could use super-anti-efficiency as the auxiliary information as discussed in subsection 2.3.
} 


$$
\partial h_{I I}^{*} / \partial h_{b}^{*}=1 / 2, \text { and } \partial h_{I I}^{*} / \partial h_{w}^{*}=1 /\left[2\left(h_{w}^{*}\right)^{2}\right]
$$

Since the range of $h_{w}^{*}$ is $[1,+\infty)$, it is clear that $\partial h_{I I}^{*} / \partial h_{w}^{*} \leq \partial h_{I I}^{*} / \partial h_{b}^{*}$. That is the marginal increase in CDI-II remains constant with respect to efficiency score, but it is diminishing with respect to anti-efficiency score.

To have more equal roles for the two distances, we may adopt the third approach to define the "CDI-III" as $h_{I I I}^{*}=\left[h_{b}^{*} \cdot h_{w}^{*}\right]^{1 / 2}$. If we use CDI-III, $h_{b}^{*}$ and $h_{w}^{*}$ can be compensated more equivalently: the score will not change when $h_{b}^{*}$ increases, but $h_{w}^{*}$ decreases in the same percentage respectively. This is not true for CDI-II.

In many scenarios, CDI-II and III are reasonable. For instance, suppose there are many homogeneous firms in one industry. Obviously, these firms on the anti-efficient frontier can be regarded as the firms with most inefficient operations (worst practices). Therefore such firms have much more risks to be driven out of market than those firms far from anti-efficient frontier. Therefore, investors will favor those firms either on the efficient frontier (leading in some aspects) or far from the anti-efficient frontier (less possibility of falling into bankruptcy). Moreover, the results of the simulation experiments in Section 4 shows that CDI-II and III are more reliable measures than the single BCC model with respect to true efficiency if the sample size is small (less than $50)$.

\subsection{Intersection of Efficient and Anti-Efficient Frontiers}

From Figure 1, we can imagine that sometimes the efficient and anti-efficient frontiers do meet. In fact this was quite normal - it means that a unit did very well in some aspects but poorly in others. This however may reduce the discrimination power of our method. In this section we will address this issue. We will discuss a sufficient condition in the appendix of this paper to ensure that the efficient and anti-efficient frontiers will not intersect so that we can make sure that our method is meaningful in a particular application. See Appendix A for details.

From Figure 1, we can imagine that sometimes the efficient and anti-efficient frontiers do meet. In fact this was quite normal - it means that a unit did very well in 
some aspects but poorly in others. This however may reduce the discrimination power of our method. In this section we will address this issue. However in real applications, often there are DMUs that are on both the efficient and anti-efficient frontiers, as shown in the following examples.

Example 1: In May 1998, the Ministry of Education of China (MOE) implemented the "Educational Revitalization Action Plan for the 21st Century", which focuses on creating world-class and high-level universities in China. This is referred as the" 985 "project. In this example, we select 7 Project 985 universities in the southeast as the DMUs to be evaluated, which are Fudan University (FDU), Tongji University (TJU), Shanghai Jiaotong University (SJTU), East China Normal University (ECNU), Nanjing University (NJU), Southeast University (SEU), and Zhejiang University (ZJU). These 7 universities are all top-level universities and located at the southeast of China, where the level of economic development is among the highest in China. The input-output data of the science and technology (S\&T) activities of these universities in 2010 are shown in Table 1 as follows.

Table 1: Input-output data of the S\&T activities of 7 universities in 2010.

\begin{tabular}{|c|c|c|c|c|c|c|c|c|}
\hline \multirow[b]{2}{*}{$\begin{array}{l}\text { Unive } \\
\text { rsities }\end{array}$} & \multicolumn{2}{|c|}{ Input indicators } & \multicolumn{4}{|c|}{ Output indicators } & \multirow{2}{*}{$\begin{array}{c}\text { CCR } \\
\text { (Model } \\
1)\end{array}$} & \multirow{2}{*}{$\begin{array}{c}\text { Inverte } \\
\text { d CCR } \\
\text { (Model } \\
4)\end{array}$} \\
\hline & $\begin{array}{l}\text { STAF } \\
\text { F } \\
\text { (Num } \\
\text { ber) }\end{array}$ & $\begin{array}{l}\text { FUND } \\
\text { (RMB in } \\
\text { thousand } \\
\text { s) }\end{array}$ & $\begin{array}{l}\text { Monog } \\
\operatorname{raph}(\mathrm{N} \\
\text { umber })\end{array}$ & $\begin{array}{c}\text { Paper } \\
\text { (Number } \\
\text { ) }\end{array}$ & $\begin{array}{l}\text { TT income } \\
\text { (RMB in } \\
\text { thousands) }\end{array}$ & $\begin{array}{c}\text { Award } \\
\text { (Numb } \\
\text { er) }\end{array}$ & & \\
\hline FDU & 7670 & 1747498 & 37 & 8615 & 43575 & 31 & 0.696 & 1 \\
\hline TJU & 5418 & 1688046 & 15 & 5717 & 2270 & 38 & 0.702 & 1 \\
\hline SJTU & 12914 & 2722304 & 99 & 19899 & 166995 & 79 & 1 & 1.383 \\
\hline $\mathrm{ECN}$ & & & & & & & 0.742 & 1 \\
\hline $\mathrm{U}$ & 1159 & 349159 & 7 & 1557 & 680 & 5 & & \\
\hline NJU & 1856 & 897794 & 14 & 5012 & 3376 & 17 & 1 & 1.046 \\
\hline SEU & 3753 & 1471192 & 40 & 6112 & 108086 & 26 & 1 & 1.021 \\
\hline ZJU & 13138 & 3290607 & 36 & 16263 & 47055 & 135 & 1 & 1 \\
\hline
\end{tabular}

Source: S \& T statistics compilation in 2011.

We can see that Zhejiang Univ. is on both the efficient and anti-efficient frontiers. Zhejiang Univ. is one of four best universities in China. The possible explanation of it's on the anti-efficient frontier is that it may have gone exceedingly to achieve its superiority in some areas, and this has brought some side effects. We can treat it as a 
normal DMU or a special unit. Here we provide two possible approaches: The first one is to treat it indifferently with other DMUs, i.e., we can use the performance indicators defined in Section 3 directly. In such a case the DMUs on both the efficient and antiefficient frontiers will have the same performance scores and cannot be discriminated. The other one is to consider it as an outlier in the evaluation and treat it differently. Therefore we apply the super anti-efficiency model to compute their anti-efficiencies. Thus we have the following procedure to have a full ranking of DMUs:

Step 1: Compute the super-anti-efficiencies of the intersected DMUs using the following super-anti-efficiency model (8):

$$
\begin{aligned}
& h_{w^{\prime}}^{*}=\operatorname{Max} \quad \theta-\varepsilon\left(\sum_{i=1}^{m} s_{i}^{-}+\sum_{r=1}^{s} s_{r}^{+}\right) \\
& \text {s.t. }\left\{\begin{array}{c}
\sum_{j=1, j \neq j_{0}}^{n} x_{i j} \lambda_{j}-s_{i}^{-}=\theta x_{i 0}, i=1, \ldots, m, \\
\sum_{j=1, j \neq j_{0}}^{n} y_{r j} \lambda_{j}+s_{r}^{+}=y_{r 0}, r=1, \ldots, s, \\
\lambda_{j}, s_{i}^{-}, s_{r}^{+} \geq 0, j=1, \ldots, n .
\end{array}\right.
\end{aligned}
$$

Step 2: Use the super-anti-efficiencies $h_{w^{\prime}}^{*}$ obtained from model (8) as the auxiliary information to further rank the DMUs on both efficient and anti-efficient frontiers for our proposed performance indicators.

The two possible solutions can be selected according to the practical needs whether a totally full ranking is needed.

\section{Empirical Studies}

In this section, we apply the three indicators to two empirical examples to show their discrimination abilities. It is concluded from the simulation analysis below that the above DEA indicators have better discrimination power when using the CCR antiefficiency DEA models. Thus we will always use the CCR anti-efficiency DEA models in our empirical studies.

As the first case, here we reconsider the Example 1 mentioned in Section 2.2. The 
efficiency and anti-efficiency scores of the universities in the input-oriented CCR model (1) and the Inverted CCR model (4) are shown in Table 1. We can see that Zhejiang Univ. is on both the efficient and the anti-efficient frontiers. Following the analysis in Section 2.3, we will use both two possible treatments on this university. Firstly, we will compute three performance indicators using its efficiency and antiefficiency directly. Secondly, we will use the super-anti-efficiency model (8) to compute its anti-efficiency. Following the procedure proposed in Subsection 2.3, we have the following steps to rank these universities.

Step 1: Compute the super-anti-efficiency of Zhejiang Univ. using the super-antiefficiency model (8). We can see its super-anti-efficiency is 0.990 .

Step 2: We also compute the three indicators. The results of the CCR model, and the CDI-I, II and III are illustrated respectively in Table 2.

Table 2: Results and ranks of 7 universities using different models.

\begin{tabular}{|c|c|c|c|c|c|c|c|c|}
\hline \multirow[b]{2}{*}{ Universities } & \multicolumn{2}{|c|}{$\mathrm{CCR}$} & \multicolumn{2}{|c|}{ CDI-I } & \multicolumn{2}{|l|}{ CDI-II } & \multicolumn{2}{|c|}{ CDI-III } \\
\hline & $h_{b}^{*}$ & $\begin{array}{c}\text { Rank } \\
1\end{array}$ & $h_{w}^{*} \cdot \varepsilon$ & $\begin{array}{c}\text { Rank } \\
2\end{array}$ & $h_{I I}^{*}$ & $\begin{array}{c}\text { Rank } \\
3\end{array}$ & $h_{I I I}^{*}$ & $\begin{array}{c}\text { Rank } \\
4\end{array}$ \\
\hline SJTU & 1 & 1 & $1.383 \varepsilon$ & 1 & 0.638 & 1 & 1.176 & 1 \\
\hline NJU & 1 & 1 & $1.046 \varepsilon$ & 2 & 0.522 & 2 & 1.023 & 2 \\
\hline SEU & 1 & 1 & $1.021 \varepsilon$ & 3 & 0.510 & 3 & 1.010 & 3 \\
\hline ZJU & 1 & 1 & $\begin{array}{c}1 \varepsilon \\
/ 0.990^{\mathrm{a}}\end{array}$ & 4 & $0.500 / 0.990^{\mathrm{b}}$ & 4 & $1 / 0.990^{c}$ & 4 \\
\hline ECNU & 0.742 & 5 & $1 \varepsilon$ & 5 & 0.371 & 5 & 0.861 & 5 \\
\hline TJU & 0.702 & 6 & $1 \varepsilon$ & 6 & 0.351 & 6 & 0.838 & 6 \\
\hline FDU & 0.696 & 7 & $1 \varepsilon$ & 7 & 0.348 & 7 & 0.834 & 7 \\
\hline
\end{tabular}

a. $1 \varepsilon / 0.990$ denotes the values obtained from anti-efficiency model (4) with $\varepsilon$ and super-antiefficiency model (8) respectively.

b. $0.500 / 0.990$ denotes the value of CDI-II (0.500) with the super-anti-efficiency $(0.990)$ as the auxiliary information.

c. $1 / 0.990$ denotes the value of CDI-III (1) with the super-anti-efficiency (0.990) as the auxiliary information.

The performance of SJTU, NJU, SEU and ZJU cannot be discriminated if we only rank the DMUs according to the efficiency scores of the CCR model, although we can easily have the full ranking of these DMUs by applying the CDI-I, II, and III. In summary, we can conclude that by utilizing the information of anti-efficient frontier, our indicators have increased the discrimination power of DEA models. We now discuss 
the main differences of the three indicators. It is clear that the CDI-I is most suitable for producing full ranks completely compliant with the classic DEA results. Essentially, the CDI-II and III are arithmetic and geometric means of the efficiency and antiefficiency scores respectively. Here we firstly visualize the two performance indicators. Without loss of generality, we set the ranges of efficiency scores and anti-efficiency scores as $[0,1]$ and $[1,10]$. Thus we have the following Figure 2 and 3, which can illustrate the features of these two indicators.

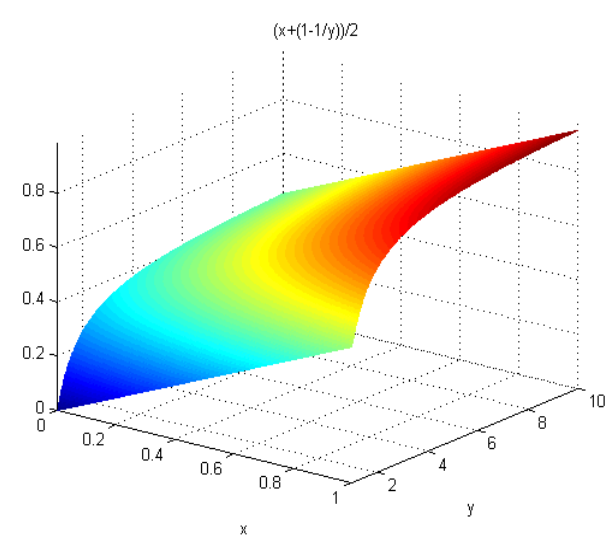

Figure 2: Visualization of CDI-II.

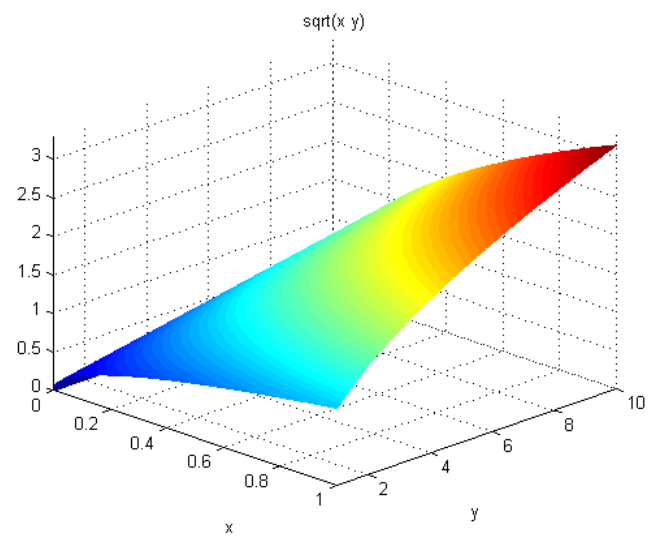

Figure 3: Visualization of CDI-III.

From Figure 2 and 3, we can see that the visual shapes of these two indicators are quite similar. In fact it is clear from the empirical results that the correlations between these two indicators are very high. However, we can see that the maximal value of CDIII is quite lower than that of CDI-III. The main reason is that the ranges of efficiency scores and anti-efficiency scores are $[0,1]$ and $[1,10]$ respectively. Based on this, we can see from these two figures that anti-efficiency scores in the CDI-III play a more important role than those in the CDI-II. Thus we can deduce that the CDI-III emphasizes anti-efficiency scores while the CDI-II emphasizes more the efficiency scores. Therefore the DMs can select the two indicators according to the real needs. In the following section we will discuss when the CDI-I or the CDI-II (CDI-III) should be used in real applications.

Next, we apply the three performance indicators on another empirical example. The data set of this example comes from Zhu (2003), which is shown in Table 3. Furthermore, we compare the results with the results using the method proposed by 
Amirteimoori (2007), which defined new combined efficiency measures based on the weighted $\mathrm{L}_{1}$-distances to both efficient and anti-efficient frontiers to rank DMUs.

Table 3: Fortune global 500 companies

\begin{tabular}{lccccc}
\hline \multirow{2}{*}{ Company } & \multicolumn{3}{c}{ Inputs } & \multicolumn{2}{c}{ Outputs } \\
\cline { 2 - 6 } & Assets & Equity & Employees & Revenue & Profit \\
\hline Mitsui & 68770.9 & 5553.9 & 80000 & 181518.7 & 314.8 \\
\hline Itochu & 65708.9 & 4271.1 & 7182 & 169164.6 & 121.2 \\
\hline General Motors & 217123.4 & 23345.5 & 709000 & 168828.6 & 6880.7 \\
\hline Sumitomo & 50268.9 & 6681 & 6193 & 167530.7 & 210.5 \\
\hline Marubeni & 71439.3 & 5239.1 & 6702 & 161057.4 & 156.6 \\
\hline Ford Motor & 243283 & 24547 & 346990 & 137137 & 4139 \\
\hline Toyota Motor & 106004.2 & 49691.6 & 146855 & 111052 & 2662.4 \\
\hline Exxon & 91296 & 40436 & 82000 & 110009 & 6470 \\
\hline Royal Dutch/Shell Group & 118011.6 & 58986.4 & 104000 & 109833.7 & 6904.6 \\
\hline Wal-Mart & 37871 & 14762 & 675000 & 93627 & 2740 \\
\hline Hitachi & 91620.9 & 29907.2 & 331852 & 84167.1 & 1468.8 \\
\hline Nippon Life Insurance & 364762.5 & 2241.9 & 89690 & 83206.7 & 2426.6 \\
\hline Nippon Telegraph \&Telephone & 127077.3 & 42240.1 & 231400 & 81937.2 & 2209.1 \\
\hline AT\&T & 88884 & 17274 & 299300 & 79609 & 139 \\
\hline
\end{tabular}

At first, we employ the input-oriented CCR model (1) and anti-efficient CCR model (4) to calculate the efficiency and anti-efficiency scores. Then we use the three DEA performance indicators. With the intention to find the characteristics and differences of these models, we ranked the DMUs according to the scores from the different models. As shown in Table 4, the ranks R1, R2, R3 and R4 are generated by the CCR efficiency score, CDI- I, CDI- II and CDI- III respectively.

It is clear that both the CCR and Inverted CCR models have weaker power of discrimination in this empirical study. Among 14 companies, 7 companies are efficient in the CCR model, and 6 companies are anti-efficient. As CDI- I used the efficiency and anti-efficiency scores of CCR and inverted CCR models $\left(h_{I}^{*}=h_{b}^{*}+h_{w}^{*} \cdot \varepsilon\right)$ with Lexicographic order to rank DMUs, it is clear that the ranks of CDI- I do not conflict with those from the CCR model. Furthermore, CDI- I can almost discriminate these efficient DMUs identified by CCR model.

Table 4: Results and ranks of companies using different models

\begin{tabular}{lllll}
\hline Company & CCR & CDI- I & CDI- II & CDI- III \\
\hline
\end{tabular}




\begin{tabular}{ccccccccc}
\hline & $h_{b}^{*}$ & Rank1 & $h_{w}^{*} \cdot \varepsilon$ & Rank2 & $h_{I I}^{*}$ & Rank3 & $h_{I I I}^{*}$ & Rank4 \\
\hline Mitsui & 1.00 & 1 & $2.93 \varepsilon$ & 1 & 0.83 & 1 & 1.71 & 1 \\
Sumitomo & 1.00 & 1 & $2.68 \varepsilon$ & 2 & 0.81 & 2 & 1.64 & 2 \\
Exxon & 1.00 & 1 & $1.45 \varepsilon$ & 3 & 0.65 & 3 & 1.20 & 4 \\
General Motors & 1.00 & 1 & $1.22 \varepsilon$ & 4 & 0.59 & 6 & 1.10 & 6 \\
Itochu & 1.00 & 1 & $1.18 \varepsilon$ & 5 & 0.58 & 7 & 1.09 & 7 \\
Wal-Mart & 1.00 & 1 & $1.00 \varepsilon$ & 6 & 0.50 & 8 & 1.00 & 8 \\
Nippon Life & 1.00 & 1 & $1.00 \varepsilon$ & 6 & 0.50 & 8 & 1.00 & 8 \\
Marubeni & 0.97 & 8 & $1.40 \varepsilon$ & 8 & 0.63 & 4 & 1.17 & 5 \\
Royal Group & 0.84 & 9 & $1.00 \varepsilon$ & 9 & 0.42 & 11 & 0.92 & 11 \\
Ford Motor & 0.74 & 10 & $1.31 \varepsilon$ & 10 & 0.49 & 10 & 0.98 & 10 \\
Toyota Motor & 0.52 & 12 & $1.07 \varepsilon$ & 12 & 0.30 & 12 & 0.75 & 12 \\
Hitachi & 0.39 & 13 & $1.00 \varepsilon$ & 13 & 0.19 & 13 & 0.62 & 13 \\
Nippon T\&T & 0.35 & 14 & $1.00 \varepsilon$ & 14 & 0.17 & 14 & 0.59 & 14 \\
AT\&T & 0.27 & 15 & $1.00 \varepsilon$ & 15 & 0.14 & 15 & 0.52 & 15 \\
\hline
\end{tabular}

It is interesting to see that the $h_{b}^{*}, h_{w}^{*}$ scores of Wal-Mart and Nippon Life Insurance both are 1 in the CCR model and Inverted CCR model, which implies that they are located on both the efficient and anti-efficient frontiers. Hence, it may not be reasonable if we only rank the DMUs according to the scores of the CCR model. However, if we apply the CDI- I, II, and III, the ranks obtained respectively are $6^{\text {th }}, 8^{\text {th }}$, and $8^{\text {th }}$, which are much lower than the ranks obtained from CCR model. The following table shows correlation among these results.

Table 5: The Spearman's correlation Coefficient of 4 ranks

\begin{tabular}{ccccc}
\hline Coefficient & Rank R1 & Rank R2 & Rank R3 & Rank R4 \\
\hline Rank R1 & 1 & & & \\
Rank R2 & $0.950^{* *}$ & 1 & & \\
Rank R3 & $0.769^{* *}$ & $0.871^{* *}$ & 1 & \\
Rank R4 & $0.731^{* *}$ & $0.832^{* *}$ & $0.989^{* *}$ & 1 \\
\hline
\end{tabular}

** Correlation is significant at the 0.01 level (2-tailed).

* Correlation is significant at the 0.05 level (2-tailed).

As shown in Table 5, the Spearman's correlation coefficient of Rank 1 and Rank 2 is 0.950 , which implies that the results form CCR and CDI-I are closely correlated as they are. Meanwhile, the CDI- II and III also show higher correlations.

If we would like to further rank the Wal-Mart and Nippon Life Insurance and have 
a full ranking of those companies, we can use follow the procedure proposed in Subsection 2.3. Therefore, we have the following results in Table 6:

Table 6: Full ranking of companies.

\begin{tabular}{lccccccccc}
\hline & \multicolumn{3}{c}{ CCR } & \multicolumn{2}{c}{ CDI- I } & \multicolumn{2}{c}{ CDI- II } & \multicolumn{2}{c}{ CDI- III } \\
\cline { 2 - 10 } Company & $h_{b}^{*}$ & Rank 1 & $h_{w}^{*} \cdot \varepsilon$ & Rank & $h_{I I}^{*}$ & Rank 3 & $h_{I I I}^{*}$ & Rank 4 \\
\hline Mitsui & 1.00 & 1 & $2.93 \varepsilon$ & 1 & 0.83 & 1 & 1.71 & 1 \\
Sumitomo & 1.00 & 1 & $2.68 \varepsilon$ & 2 & 0.81 & 2 & 1.64 & 2 \\
Exxon & 1.00 & 1 & $1.45 \varepsilon$ & 3 & 0.65 & 3 & 1.20 & 4 \\
General & 1.00 & 1 & $1.22 \varepsilon$ & 4 & 0.59 & 6 & 1.10 & 6 \\
Motors & 1.00 & 1 & $1.18 \varepsilon$ & 5 & 0.58 & 7 & 1.09 & 7 \\
Itochu & 1.00 & 1 & $1.00 \varepsilon$ & 6 & $0.50 / 0.56$ & 8 & $1.00 / 0.56$ & 8 \\
& & & 10.56 & & & & & \\
Wal-Mart & 1.00 & 1 & $1.00 \varepsilon$ & 7 & $0.50 / 0.40$ & 9 & $1.00 / 0.40$ & 9 \\
& & & 10.40 & & & & & \\
Nippon Life & 0.97 & 8 & $1.40 \varepsilon$ & 8 & 0.63 & 4 & 1.17 & 5 \\
Marubeni & 0.84 & 9 & $1.00 \varepsilon$ & 9 & 0.42 & 11 & 0.92 & 11 \\
Royal Group & 0.74 & 10 & $1.31 \varepsilon$ & 10 & 0.49 & 10 & 0.98 & 10 \\
Ford Motor & 0.52 & 12 & $1.07 \varepsilon$ & 12 & 0.30 & 12 & 0.75 & 12 \\
Toyota Motor & 0.39 & 13 & $1.00 \varepsilon$ & 13 & 0.19 & 13 & 0.62 & 13 \\
Hitachi & 0.35 & 14 & $1.00 \varepsilon$ & 14 & 0.17 & 14 & 0.59 & 14 \\
Nippon T\&T & 0.27 & 15 & $1.00 \varepsilon$ & 15 & 0.14 & 15 & 0.52 & 15 \\
AT\&T & & & & & & & &
\end{tabular}

From Table 6 we can see we have discriminate the two DMUs (Wal-Mart and Nippon Life Insurance) and have a full ranking of all DMUs.

Furthermore, we compare the above results in Table 6 with the results of the method proposed by Amirteimoori (2007). The results are shown in the following Table 7. From this table, we can see that, although Amirteimoori (2007) proposed interesting new combined efficiency measures based on the weighted $\mathrm{L}_{1}$-distances, there still are some drawbacks: (1) the new efficiency measure has low discrimination in the sense that it cannot rank fully the DMUs to be evaluated. For example, using the efficiency measure $\mathrm{e}_{0}^{*}$, the scores of all efficient DMUs are 1 and the scores of all full-inefficient DMUs are all -1. (2) The super-efficiency measure $\mathrm{se}_{0}^{*}$ may suffer infeasibility problem. (3) The DMUs on the intersection of both efficient and anti-efficient frontiers are measured as -1 in either efficiency measure $\mathrm{e}_{0}^{*}$ or the super-efficiency measure $\mathrm{e}_{0}^{*}$, i.e., the super-efficiency $\mathrm{se}_{0}^{*}$ in Amirteimoori (2007) cannot discriminate the full-in-efficient 
DMUs on anti-efficient frontier.

Table 7. Comparison of different measures.

\begin{tabular}{lllc}
\hline Company & \multicolumn{1}{c}{$\mathrm{u}_{0}^{+}$} & \multicolumn{1}{c}{$\mathrm{u}_{0}^{-}$} & $\begin{array}{c}\text { Efficiency measure } \mathrm{e}_{0}^{*} \text { in } \\
\text { Amirteimoori (2007) }\end{array}$ \\
\hline Mitsui & 0.0000 & 370178.3373 & 1.0000 \\
Itochu & 0.0000 & 34261.7511 & 1.0000 \\
General Motors & 0.0000 & 200222.7829 & 1.0000 \\
Sumitomo & 0.0000 & 113049.5835 & 1.0000 \\
Marubeni & 13701.1234 & 52047.3682 & 0.5832 \\
Ford Motor & 197030.5311 & 164844.1294 & -0.0889 \\
Toyota Motor & 76620.3828 & 126479.3805 & 0.2455 \\
Exxon & 0.0000 & 175864.5245 & 1.0000 \\
Royal Dutch/Shell Group & 18072.0558 & 0.0000 & -1.0000 \\
Wal-Mart & 0.0000 & 0.0000 & 1.0000 \\
Hitachi & 240457.6222 & 0.0000 & -1.0000 \\
Nippon Life Insurance & 0.0000 & 0.0000 & 1.0000 \\
Nippon Telegraph & $\&$ & & -1.0000 \\
Telephone & 146104.7503 & 0.0000 & -1.0000 \\
AT\&T & 233171.1797 & 0.0000 & \\
\hline
\end{tabular}

\section{Simulation Study}

To further examine the effectiveness of our indicators, we design three Monte Carlo experiments in this section. In each experiment, we firstly generate data from a known data generating process as described in Section 4.1, and then conduct 2000 Monte Carlo trails.

\subsection{Data Generating Process}

Although traditionally DEA was regarded as non-statistical approach, Banker and Natarajan (2008) provided a coherent Data Generating Process (DGP) for the two-stage analysis based on DEA. Following the line of Banker and Natarajan (2008), we employ two similar data generating processes in our simulation study. At first, we assume that the inputs and output data of DMUs are generated from the true production function $\phi(x)$ and an error term $\delta$. The production function $\phi(x)$ is monotone increasing and concave with respect to inputs $X$. Thus, we can specify the following equation to relate 
the inputs vector $X$ to a single output $y$ :

$$
y=\phi(x) \times e^{\delta},
$$

Then we assume that the error term $\delta$ is composed of a one-sided distributed technical inefficiency $u$ and a two sided distributed random noise $v$, that is $\delta=v-u$. The specification of error term $\delta$ is analogous to composed error formulations in parametric stochastic frontier models.

In our study, the technical inefficiency $u$ is drawn from a half normal distribution, that is $u \sim\left|N\left(0, \sigma_{u}^{2}\right)\right|$. And the parameter $\sigma_{u}^{2}$ is generated from a uniform distribution over the interval $[0,0.1998]$ and so the mean efficiency given by $E(\theta)=$ $\exp \left(-\sigma_{u} \sqrt{2 / \pi}\right)$ is between 0.7 and 1. Meanwhile, the random noise $v$ is generated from a two-sided truncated normal distribution $N\left(0, \sigma_{v}^{2}\right)$ with upper and lower bounds at $6 \sigma_{v}$ and $-6 \sigma_{v}$. The $\sigma_{v}$ is sampled from a uniform distribution over the interval $[0.02,0.06]$.

Next we will separately use different specifications of true production function $\phi(x)$ to conduct three simulation experiments as described from Section 4.2 to 4.4.

\subsection{Simulation Experiment I}

Banker and Chang (2006) employed the following "shifted" Cobb-Douglas function in their simulation study:

$$
\phi(x)=\prod_{i=1}^{m}\left(x_{i}-\alpha_{i}\right)^{\beta_{i}},
$$

where $\alpha_{i}=5$, the inputs $x_{i}$ are drawn randomly from independent uniform distributions on the interval $[10,20]$. In our simulation study, if the inputs are two, then the coefficients $\beta_{1}$ and $\beta_{2}$ are generated randomly from independent uniform distributions on the interval $[0.4,0.5]$; Otherwise if the inputs are three, then the coefficients $\beta_{1}$ and $\beta_{2}$ are generated randomly from independent uniform distributions on the interval $[0.2,0.3]$, while $\beta_{3}$ is sampled randomly from 
independent uniform distributions on the interval $[0.2,0.4]$. Because the sum of $\beta_{i}$ is less than one, the production function $\phi(x)$ is concave and satisfies the same assumption of variable return to scale as BCC DEA model.

Next, we need to generate $N$ observations for each Monte Carlo trial. First, we randomly draw an integer value for $N$ and values for $\sigma_{u}, \sigma_{v}$ and $\beta_{i}$. Then we randomly generate $N$ observations of the inputs $x_{i k}$, and the true efficiency $\theta_{k}=e^{-u_{k}}$, $k=1, \ldots, N$. Finally, we obtain the observed output $y_{k}$ by the production function (10). In this manner we obtain a sample which contains $N$ observations $\left(y_{k}, x_{1 k}, \ldots, x_{m k}\right)$.

\subsection{Simulation Experiment II}

Simar and Wilson (2007) employed the following function to represent a concave and monotone increasing production function $\phi(x)$ :

$$
\phi(x)=\sum_{i=1}^{m} x_{i}^{\beta_{i}}
$$

where $\beta_{i}=3 / 4$. However, we generate $\beta_{i}$ from independent uniform distributions on the interval $[0.6,1]$ to get more generalized simulation results. The inputs $x_{i}$ are drawn randomly from independent uniform distributions on the interval $[6,16]$.

Finally, we generate $N$ observations for each Monte Carlo trial following the same procedures described in Section 4.2.

\subsection{Simulation Experiment III}

Banker and Natarajan (2008) used the following cubic polynomial to represent the production technology $\phi(x)$ :

$$
\phi(x)=\sum_{i=1}^{m}\left(a_{0}+a_{1} x_{i}+a_{2} x_{i}^{2}+a_{3} x_{i}^{3}\right)
$$

where the coefficients $a_{0}, a_{1}, a_{2}$ and $a_{3}$ determine the properties of the production technology $\phi(x)$. Here, we employ the same setting $\left(a_{0}=-37, a_{1}=48, a_{2}=-12\right.$, 
$\left.a_{3}=1\right)$ as in Banker and Natarajan (2008) so that $\phi(x)$ is continuous, monotone increasing and concave in range [1, 4]. Then we generate the input variables $x_{i}$ from independent uniform distributions over the interval [1,4] independently.

Finally, we generate $N$ observations for each Monte Carlo trial following the same procedures described in Section 4.2.

\subsection{Sample Size}

To test the performance of our indicators, we carry out each type of simulation experiments separately as the samples vary from small to large. That is we consider a sample of size $N$, and for each of the three experiments, we repeatedly conduct the simulation experiment five times, but the sample size $N$ takes any integer value on the intervals $[5,20],[20,50],[50,200]$, and $[200,500]$ with equal probability respectively.

Then we run each simulation experiments for two thousand times independently.

\subsection{Results of the Experiments}

As the production function assumed above is concave, it seems very natural to apply the BCC model to estimate the production function. Hence, as for the anti-efficient frontier, we employ both the inverted CCR and BCC models to estimate the antiefficient frontiers for comparisons. The averages of Pearson and Spearman correlation coefficients of 2000 experiments are presented in Table 8 and 9. 
Table 8: The averages of Pearson correlation coefficients between true efficiency and the results of BCC, Inverted CCR, CDI- II and CDI- III

\begin{tabular}{|c|c|c|c|c|c|c|c|c|c|}
\hline \multirow{2}{*}{$\begin{array}{c}\text { Types of } \\
\text { Experiment }\end{array}$} & \multirow{2}{*}{$\begin{array}{c}\text { Corre. } \\
\text { Coefficient }\end{array}$} & \multicolumn{2}{|c|}{$\mathrm{N} \in[5,20]$} & \multicolumn{2}{|c|}{$\mathrm{N} \in[20,50]$} & \multicolumn{2}{|c|}{$N \in[50,200]$} & \multicolumn{2}{|c|}{$\mathrm{N} \in[200,500]$} \\
\hline & & $m=2$ & $m=3$ & $m=2$ & $m=3$ & $m=2$ & $m=3$ & $m=2$ & $m=3$ \\
\hline \multirow{7}{*}{$\begin{array}{c}\text { Experiment } \\
\text { I }\end{array}$} & $\mathrm{BCC}$ & 0.6616 & 0.5639 & 0.7982 & 0.6702 & 0.8704 & 0.7334 & 0.8986 & 0.7625 \\
\hline & Inv. BCC & 0.5378 & 0.5019 & 0.6282 & 0.5726 & 0.6928 & 0.6272 & 0.7282 & 0.6580 \\
\hline & CDI-II-B & 0.7262 & 0.6199 & 0.8199 & 0.6920 & 0.8648 & 0.7372 & 0.8815 & 0.7587 \\
\hline & CDI-III-B & 0.7190 & 0.6168 & 0.8098 & 0.6874 & 0.8553 & 0.7309 & 0.8728 & 0.7508 \\
\hline & Inv. CCR & 0.7904 & 0.6946 & 0.8254 & 0.7167 & 0.8422 & 0.7342 & 0.8466 & 0.7362 \\
\hline & CDI-II-C & 0.8494 & 0.7065 & 0.8942 & 0.7476 & 0.9160 & 0.7774 & 0.9209 & 0.7878 \\
\hline & CDI-III-C & 0.8493 & 0.7079 & 0.8948 & 0.7446 & 0.9143 & 0.7694 & 0.9172 & 0.7766 \\
\hline \multirow{7}{*}{$\begin{array}{c}\text { Experiment } \\
\text { II }\end{array}$} & $\mathrm{BCC}$ & 0.6658 & 0.6102 & 0.7857 & 0.7063 & 0.8623 & 0.7623 & 0.8977 & 0.7969 \\
\hline & Inv. BCC & 0.5471 & 0.5155 & 0.6425 & 0.5931 & 0.7014 & 0.6450 & 0.7426 & 0.6839 \\
\hline & CDI-II-B & 0.7328 & 0.6585 & 0.8192 & 0.7323 & 0.8632 & 0.7705 & 0.8857 & 0.7980 \\
\hline & CDI-III-B & 0.7264 & 0.6529 & 0.8111 & 0.7246 & 0.8552 & 0.7616 & 0.8783 & 0.7881 \\
\hline & Inv. CCR & 0.7769 & 0.6538 & 0.8144 & 0.6884 & 0.8383 & 0.7003 & 0.8497 & 0.7099 \\
\hline & CDI-II-C & 0.8220 & 0.7118 & 0.8689 & 0.7661 & 0.8997 & 0.7929 & 0.9147 & 0.8135 \\
\hline & CDI-III-C & 0.8227 & 0.7042 & 0.8687 & 0.7521 & 0.8969 & 0.7743 & 0.9099 & 0.7915 \\
\hline \multirow{7}{*}{$\begin{array}{c}\text { Experiment } \\
\text { III }\end{array}$} & $\mathrm{BCC}$ & 0.6674 & 0.5190 & 0.7890 & 0.6055 & 0.8665 & 0.6647 & 0.8972 & 0.6833 \\
\hline & Inv. BCC & 0.4249 & 0.4166 & 0.5025 & 0.4644 & 0.5382 & 0.4994 & 0.5521 & 0.5106 \\
\hline & CDI-II-B & 0.6300 & 0.5444 & 0.7211 & 0.5980 & 0.7647 & 0.6306 & 0.7823 & 0.6400 \\
\hline & CDI-III-B & 0.6134 & 0.5387 & 0.6991 & 0.5905 & 0.7368 & 0.6216 & 0.7471 & 0.6273 \\
\hline & Inv. CCR & 0.6104 & 0.5490 & 0.6350 & 0.5717 & 0.6193 & 0.5885 & 0.6001 & 0.5879 \\
\hline & CDI-II-C & 0.7402 & 0.6096 & 0.8095 & 0.6500 & 0.8460 & 0.6770 & 0.8644 & 0.6805 \\
\hline & CDI-III-C & 0.7280 & 0.6055 & 0.7869 & 0.6433 & 0.7978 & 0.6669 & 0.7891 & 0.6678 \\
\hline
\end{tabular}


Table 9: The averages of Spearman correlation coefficients between true efficiency and the results of BCC, Inverted CCR, CDI-I, II and III

\begin{tabular}{|c|c|c|c|c|c|c|c|c|c|}
\hline \multirow{2}{*}{$\begin{array}{c}\text { Types of } \\
\text { Experiment }\end{array}$} & \multirow{2}{*}{$\begin{array}{c}\text { Corre. } \\
\text { Coefficient }\end{array}$} & \multicolumn{2}{|c|}{$\mathrm{N} \in[5,20]$} & \multicolumn{2}{|c|}{$\mathrm{N} \in[20,50]$} & \multicolumn{2}{|c|}{$\mathrm{N} \in[50,200]$} & \multicolumn{2}{|c|}{$\mathrm{N} \in[200,500]$} \\
\hline & & $m=2$ & $m=3$ & $m=2$ & $m=3$ & $m=2$ & $m=3$ & $m=2$ & $m=3$ \\
\hline \multirow{5}{*}{$\begin{array}{c}\text { Experiment } \\
\text { I }\end{array}$} & $\mathrm{BCC}$ & 0.5957 & 0.4922 & 0.7358 & 0.6154 & 0.8376 & 0.7059 & 0.8824 & 0.7463 \\
\hline & Inv. CCR & 0.7560 & 0.6640 & 0.8041 & 0.6987 & 0.8262 & 0.7200 & 0.8323 & 0.7229 \\
\hline & CDI-I & 0.7238 & 0.5783 & 0.7807 & 0.6432 & 0.8480 & 0.7122 & 0.8846 & 0.7476 \\
\hline & CDI-II & 0.8063 & 0.6553 & 0.8726 & 0.7072 & 0.9022 & 0.7436 & 0.9105 & 0.7585 \\
\hline & CDI-III & 0.8050 & 0.6575 & 0.8705 & 0.7118 & 0.8993 & 0.7471 & 0.9058 & 0.7588 \\
\hline \multirow{5}{*}{$\begin{array}{c}\text { Experiment } \\
\text { II }\end{array}$} & $\mathrm{BCC}$ & 0.5964 & 0.5358 & 0.7224 & 0.6453 & 0.8307 & 0.7353 & 0.8824 & 0.7834 \\
\hline & Inv. CCR & 0.7516 & 0.6377 & 0.7979 & 0.6840 & 0.8251 & 0.7007 & 0.8383 & 0.7125 \\
\hline & CDI-I & 0.7007 & 0.5994 & 0.7593 & 0.6666 & 0.8387 & 0.7397 & 0.8841 & 0.7843 \\
\hline & CDI-II & 0.7798 & 0.6578 & 0.8416 & 0.7216 & 0.8809 & 0.7616 & 0.9016 & 0.7889 \\
\hline & CDI-III & 0.7801 & 0.6587 & 0.8430 & 0.7234 & 0.8806 & 0.7603 & 0.8984 & 0.7832 \\
\hline \multirow{5}{*}{$\begin{array}{c}\text { Experiment } \\
\text { III }\end{array}$} & $\mathrm{BCC}$ & 0.6112 & 0.4724 & 0.7275 & 0.5627 & 0.8289 & 0.6422 & 0.8753 & 0.6726 \\
\hline & Inv. CCR & 0.5792 & 0.5241 & 0.6176 & 0.5536 & 0.6057 & 0.5738 & 0.5877 & 0.5739 \\
\hline & CDI-I & 0.6935 & 0.5350 & 0.7764 & 0.5916 & 0.8439 & 0.6499 & 0.8792 & 0.6745 \\
\hline & CDI-II & 0.6880 & 0.5639 & 0.7855 & 0.6200 & 0.8397 & 0.6586 & 0.8650 & 0.6698 \\
\hline & CDI-III & 0.6779 & 0.5612 & 0.7574 & 0.6140 & 0.7788 & 0.6468 & 0.7751 & 0.6525 \\
\hline
\end{tabular}

Note: 1 . when $\mathrm{N} \in[5,20]$, among 2000 trials there are 23, 19 and 53 trials that all the DMUs generated are BCC efficient for three types of experiment respectively.

2. In Table 8, CDI-II-B, CDI-III-B, CDI-II-C and CDI-III-C refer to the CDI-II and CDI-III based on the Inverted BCC and Inverted CCR model respectively. While in Table 9, we only show the CDI-I, CDI-II, CDI-III based on inverted CCR model.

3. We also conducted another three simulation experiments, when the error term $\varepsilon$ only consists of technical inefficiency $u$. This specification of error term is adopted by Banker (1993) and Banker and Chang (2006). And the simulation results are similar with what we have reported in Table 8 and 9. 
As Table 8 and 9 show, the averages of both the Pearson and Spearman correlation coefficients between true efficiency and our performance indicators based on the Inverted CCR model are higher than those of true efficiency and efficiency scores estimated by the BCC model in all the experiments when sample size is less than 200. Therefore, it is clear that the performance of our CDI is more satisfactory than that of BCC model, especially when the sample size is small (e.g. less than 20). These simulation results confirmed our arguments described in the beginning: for a small sample (No. of DMUs), the standard DEA models do not work well. In this case, additional information from anti-efficient frontier can increase the performance of DEA estimators.

Meanwhile, we find that the averages of correlation coefficients based on Inverted CCR are much higher than those based on Inverted BCC in all experiments. Actually, these results are not coincidence. It is more reliable to use anti-efficient CCR than antiefficient BCC, no matter whether the CCR or BCC model is employed to estimate the efficient frontier. To illustrate the difference between the two Inverted DEA models, we simply assume that there is only one input and output. The true efficient frontier and the frontiers generated by BCC, Inverted BCC and Inverted CCR are illustrated in Figure 4. The anti-efficient BCC frontier is convex, while the true efficient frontier is concave. And this phenomenon may make the DMUs with extreme inputs become relatively closer to the anti-efficiency frontier regardless whether they are close to the true frontier or not. On the contrary, the anti-efficient frontier estimated by Inverted CCR model reduces this problem. Therefore, we can conclude that the frontier of the anti-efficient CCR should be more reliable. 


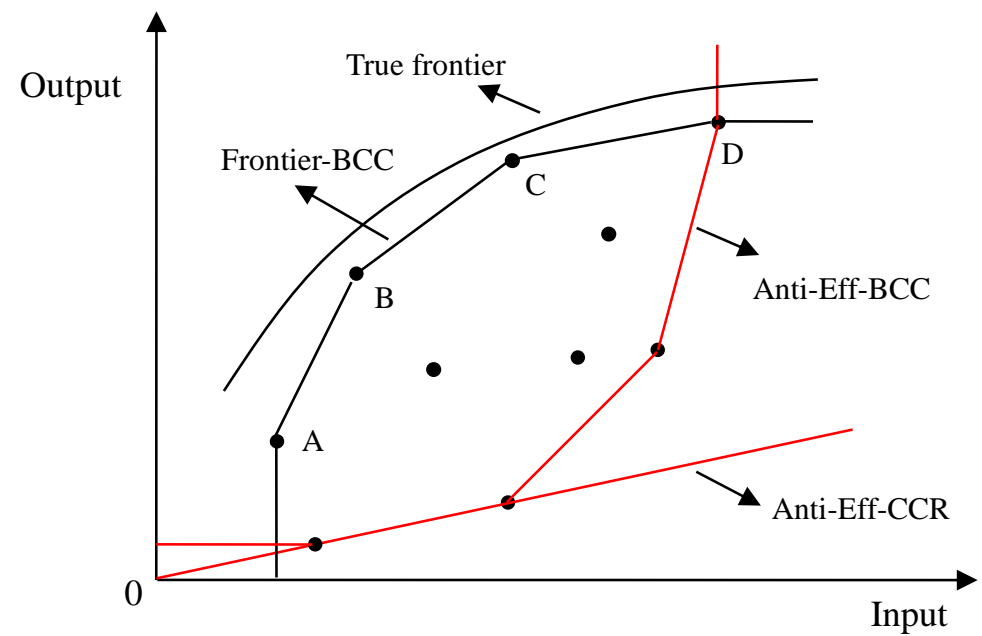

Figure 4: The anti-efficient frontiers of Inverted CCR and BCC models

As Figure 4 shows, the DMUs A, B, C and D are identified as DEA efficient by BCC model. However, they may have different true efficiency scores if the true efficient frontier is known. That is why we try to use the anti-efficient CCR frontier to discriminate these DMUs: given inputs $X$, the farther a DMU from the anti-efficient CCR frontier, the more likelihood it obtains a larger score of true efficiency. Therefore, anti-efficient frontier estimated by Inverted CCR model is able to provide us with very useful extra information to evaluate the performance of a DMU, especially when the sample is relatively small. However, as the sample size goes to infinite, the BCC frontier will be very close to the true efficient frontier (Banker 1993). Then the additional information from anti-efficient frontier becomes less useful.

Finally, as Table 9 shows, the averages of Spearman correlation coefficients of CDII are higher than those of BCC model in all experiments. However, when sample size is small (e.g. less than 50), the averages of Spearman correlation coefficients of CDI-I is lower than those of CDI-II and III. Therefore, we can conclude that it is more plausible for us to use either CDI-II or III. But as sample size goes large, it is more suitable to employ CDI-I.

\section{Conclusions}

In summary, our study shows that it is plausible to consider both the efficient and anti-efficient frontiers in DEA analysis when there are many input and output variables, 
but only few DMUs are available. The extra information of anti-efficient frontier is able to increase the discrimination power of DEA analysis quite well.

More specifically, to examine our research idea, we firstly constructed three intuitive DEA performance indicators based on the distances to both efficient and antiefficient frontier. In the two empirical examples, the empirical results show that our approach indeed increases discrimination power of DEA analysis. In the following simulation study, we further found that the averages of both Pearson and Spearman correlation coefficients between true efficiency and the three indicators are higher than those of true efficiency and efficiency scores estimated by the BCC model in all the experiments when sample size is less than 50. This performance gap is considerably large especially when the sample size is small (e.g. less than 20). Furthermore, we also found it is much better to employ Inverted CCR model to construct the performance indicators regardless which production technologies we assume. Finally, it is more plausible for us to use either CDI-II or III when sample is small. But as sample size goes large, it is more suitable to employ BCC model or CDI-I.

Acknowledgements. We acknowledge the support of the National Natural Science Foundation of China (No. 71201158).

\section{References}

[1] Adler, N., Friedman, L., Sinuany-Stern, Z. (2002) Review of ranking methods in the data envelopment analysis context, European Journal of Operational Research 140, 249-265.

[2] Allen, R., Athanassopoulos, A., Dyson, R.G., Thanassoulis, E. (1997) Weights restrictions and value judgments in data envelopment analysis: Evolution, development and future directions. Annals of Operations Research 73, 13-34.

[3] Amirteimoori, A. (2007) DEA efficiency analysis: Efficient and anti-efficient frontier, Applied Mathematics and Computation 186, 10-16.

[4] Andersen, P., Petersen, N. (1993) A procedure for ranking efficient units in data envelopment analysis, Management Science39, 1261-1264.

[5] Banker, R.D., Gifford, J.L. (1988) A relative efficiency model for the evaluation of public health nurse productivity, Mellon University Mimeo, Carnegie. 
[6] Banker, R.D. (1993) Maximum likelihood, consistency and data envelopment analysis: A statistical foundation. Management Science 39, 1265-1273.

[7] Banker, R.D., Chang, H. (2006) The super-efficiency procedure for outlier identification, not for ranking efficient units, European Journal of Operational Research 175, 1311-1320.

[8] Banker, R.D., Natarajan, R. (2008) Evaluating contextual variables affecting productivity using data envelopment analysis, Operations Research 56, 48-58.

[9] Beasley, J.E. (2003) Allocating fixed costs and resources via data envelopment analysis, European Journal of Operational Research 147, 198-216.

[10] Charnes, A., Cooper, W.W., Rhodes, E. (1978) Measuring the efficiency of decision making units, European Journal of Operational Research 2, 429-444.

[11] Chen, Y. (2004) Ranking efficient units in DEA, Omega 32,213-219.

[12] Cooper, W.W., Seiford, L.M., Tone, K. (2000) Data Envelopment Analysis: A Comprehensive Text with Models- Applications, References and DEA-Solver Software, Kluwer Academic Publishers, Boston.

[13] Dantzig, G.B. (1963) Linear Programming and Extensions. Princeton University Press, New York.

[14] Doyle, J.R., Green, R. (1994) Efficiency and cross-efficiency in data envelopment analysis: Derivatives, meanings and uses, Journal of the Operational Research Society 45(5), 567-578.

[15] Entani, T., Maeda, Y., Tanaka, H. (2002) Dual models of interval DEA and its extension to interval data, European Journal of Operational Research 136, 32-45.

[16] Hwang, C.L., Yoon, K. (1981) Multiple Attribute Decision Making: Methods and Applications, Springer-Verlag, New York.

[17] Johnson, A.L., McGinnis, L.F. (2008) Outlier detection in two-stage semiparametric DEA models, European Journal of Operational Research 187, 629-635.

[18] Liu, W.B., Meng, W., Zhang, D.Q. (2006) Chapter 21: Incorporating value judgments in DEA, In Productivity Analysis in the Service Sector using Data Envelopment Analysis - 3rd edition (Edited by Avkiran N. K.), 217-242.

[19] Liu, W.B., Meng, W., Li, X.X., Zhang, D.Q. (2010) DEA Models with Undesirable Inputs and Outputs, Annals of Operations Research173, 177-194.

[20] Liu, W.B., Zhang, D.Q., Meng, W., Li, X.X., Xu, F. (2011). A study of DEA models without explicit inputs. Omega, The International Journal of Management Science 39, 472-480.

[21] Meng W, Liu WB, Li. XX. (2005). Application of index DEA model on performance evaluation of Basic Research Institute. Science of Science and Management of Science and Technology 9, 11-6. (in Chinese).

[22] Meng,W., Zhang, D.Q., Qi, L., Liu, W.B. (2008) Two-level DEA approaches in research evaluation. Omega 36 (6), 950-957. 
[23] Paradi, J.C., Asmild, M., Simak P.C. (2004) Using DEA and Worst Practice DEA in Credit Risk Evaluation. Journal of Productivity Analysis 21, 153-165.

[24] Sexton, T.R., Silkman, R.H., Hogan, A.J. (1986) Data envelopment analysis: Critique and extensions. In: Silkman, R.H. (Ed.), Measuring Efficiency: An Assessment of Data Envelopment Analysis, Jossey-Bass, San Francisco, CA, 73-105.

[25] Simar, L., Wilson, P.W. (2007) Estimation and inference in two-stage, semi-parametric models of production processes, Journal of Econometrics 136, 31-64.

[26] Takamura, Y., Tone, K. (2003) A comparative site evaluation study for relocating Japanese government agencies out of Tokyo, Socio-Economic Planning Sciences 37, 85-102.

[27] Thanassoulis, E. (1999) Setting Achievements Targets for School Children, Education Economics 7(2), 101-119.

[28] Thanassoulis, E., Portela, M.C., Allen, R. (2004) Incorporating value judgment in DEA, In Handbook on Data Envelopment Analysis (Edited by Cooper, W.W., Seiford, L.M. and Zhu, J.), Kluwer Academic Publishers, 99-138.

[29] Wang, Y.M., Luo, Y. (2006) DEA efficiency assessment using ideal and anti-ideal decision making units, Applied Mathematics and Computation 173, 902-915.

[30] Wu, D. (2006) A note on DEA efficiency assessment using ideal point: An improvement of Wang and Luo's model, Applied Mathematics and Computation 183, 819-830.

[31] Yamada, Y., Matsui, T., Sugiyama, M. (1994) An inefficiency measurement method for management systems, Journal of the Operations Research Society of Japan 37, 158-67.

[32] Zhang, D.Q., Li, X.X., Meng, W., Liu, W.B.(2009) Measure the performance of nations at Olympic Games using DEA models with different preferences. Journal of the Operational Research Society 60, 983-990.

[33] Zhou, P., Poh, K., Ang, B. (2007) A non-radial DEA approach to measuring environmental performance. European Journal of Operational Research 178, 1-9.

[34] Zhu, J. (2003) Quantitative Models for Performance Evaluation and Benchmarking: DEA with Spreadsheets and DEA Excel Solver, Kluwer Academic Publishers, Boston. 


\section{Appendix A: A sufficient condition for the intersection of the efficient and anti-}

\section{efficient frontiers}

Here we discuss a sufficient condition theoretically to ensure that the efficient and anti-efficient frontiers will not intersect. By model (1) and model (4), we can identify the $s_{1}$ efficient DMUs, denoted by $\left(\begin{array}{c}X_{1}^{e} \\ Y_{1}^{e}\end{array}\right),\left(\begin{array}{c}X_{2}^{e} \\ Y_{2}^{e}\end{array}\right), \ldots,\left(\begin{array}{c}X_{s_{1}}^{e} \\ Y_{s_{1}}^{e}\end{array}\right)$, and the $s_{2}$ anti-efficient DMUs, denoted by $\left(\begin{array}{c}X_{1}^{a} \\ Y_{1}^{a}\end{array}\right),\left(\begin{array}{c}X_{2}^{a} \\ Y_{2}^{a}\end{array}\right), \ldots,\left(\begin{array}{c}X_{s_{1}}^{a} \\ Y_{s_{1}}^{a}\end{array}\right)$. We let $\Phi$ and $\Pi$ represent the convex combinations of the $s_{1}$ efficient DMUs and the $s_{2}$ anti-efficient DMUs respectively, i.e:

$$
\begin{gathered}
\left\{\begin{array}{c}
\lambda_{1}\left(\begin{array}{c}
X_{1}^{e} \\
Y_{1}^{e}
\end{array}\right)+\lambda_{2}\left(\begin{array}{c}
X_{2}^{e} \\
Y_{2}^{e}
\end{array}\right)+\ldots+\lambda_{s_{1}}\left(\begin{array}{c}
X_{s_{1}}^{e} \\
Y_{s_{1}}^{e}
\end{array}\right) @ \Phi \\
\lambda_{1}+\lambda_{2}+\ldots+\lambda_{s_{1}}=1 \\
\lambda_{1}, \lambda_{2}, \ldots, \lambda_{s_{1}} \geq 0
\end{array}\right. \\
\left\{\begin{array}{l}
\lambda_{1}^{\prime}\left(\begin{array}{c}
X_{1}^{a} \\
Y_{1}^{a}
\end{array}\right)+\lambda_{2}^{\prime}\left(\begin{array}{c}
X_{2}^{a} \\
Y_{2}^{a}
\end{array}\right)+\ldots+\lambda_{s_{2}}^{\prime}\left(\begin{array}{c}
X_{s_{2}}^{a} \\
Y_{s_{2}}^{a}
\end{array}\right) @ \Pi \\
\lambda_{1}^{\prime}+\lambda_{2}^{\prime}+\ldots+\lambda_{s_{2}}^{\prime}=1 \\
\lambda_{1}^{\prime}, \lambda_{2}^{\prime}, \ldots, \lambda_{s_{2}}^{\prime} \geq 0
\end{array}\right.
\end{gathered}
$$

We let $E F_{e}$ and $E F_{a}$ denote the efficient and anti-efficient frontiers respectively. Thus we have $E F_{e} \subset \Phi$ and $E F_{a} \subset \Pi$. It is clear that if there are no intersections between $\Phi$ and $\Pi$, then $E F_{e}$ and $E F_{a}$ must not intersect. Therefore we can have the following sufficient condition that can ensure there are no intersections between $\Phi$ and $\Pi$. We consider the following system of linear inequalities:

$$
\left\{\begin{array}{l}
\lambda_{1}\left(\begin{array}{l}
X_{1}^{e} \\
Y_{1}^{e}
\end{array}\right)+\lambda_{2}\left(\begin{array}{c}
X_{2}^{e} \\
Y_{2}^{e}
\end{array}\right)+\ldots+\lambda_{s_{1}}\left(\begin{array}{c}
X_{s_{1}}^{e} \\
Y_{s_{1}}^{e}
\end{array}\right)-\lambda_{1}^{\prime}\left(\begin{array}{c}
X_{1}^{a} \\
Y_{1}^{a}
\end{array}\right)-\lambda_{2}^{\prime}\left(\begin{array}{c}
X_{2}^{a} \\
Y_{2}^{a}
\end{array}\right)-\ldots-\lambda_{s_{2}}^{\prime}\left(\begin{array}{c}
X_{s_{2}}^{a} \\
Y_{s_{2}}^{a}
\end{array}\right)=0 \\
\lambda_{1}+\lambda_{2}+\ldots+\lambda_{s_{1}}=1 \\
\lambda_{1}^{\prime}+\lambda_{2}^{\prime}+\ldots+\lambda_{s_{2}}^{\prime}=1 \\
\lambda_{1}, \lambda_{2}, \ldots, \lambda_{s_{1}} \geq 0 ; \lambda_{1}^{\prime}, \lambda_{2}^{\prime}, \ldots, \lambda_{s_{2}}^{\prime} \geq 0
\end{array}\right.
$$


where $\lambda_{1}, \ldots, \lambda_{s_{1}}, \lambda_{1}^{\prime}, \ldots, \lambda_{s_{2}}^{\prime}$ are coefficients.

If there is no feasible solution in (A-3), we can ensure that the efficient and antiefficient frontiers will not intersect.

It is clear that we can introduce the following auxiliary linear programming model (A-4) with the slacks variables to determine whether or not there is feasible solution in (A-3) (see Dantzig, 1963).

$$
\begin{aligned}
& \min \rho=\sum_{i=1}^{m+s+2} v_{i} \\
& \text { s.t. }\left\{\begin{array}{l}
\lambda_{1}\left(\begin{array}{l}
X_{1}^{e} \\
Y_{1}^{e}
\end{array}\right)+\lambda_{2}\left(\begin{array}{l}
X_{2}^{e} \\
Y_{2}^{e}
\end{array}\right)+\ldots+\lambda_{s_{1}}\left(\begin{array}{c}
X_{s_{1}}^{e} \\
Y_{s_{1}}^{e}
\end{array}\right)-\lambda_{1}^{\prime}\left(\begin{array}{l}
X_{1}^{a} \\
Y_{1}^{a}
\end{array}\right)-\lambda_{2}^{\prime}\left(\begin{array}{l}
X_{2}^{a} \\
Y_{2}^{a}
\end{array}\right)-\ldots-\lambda_{s_{2}}^{\prime}\left(\begin{array}{c}
X_{s_{2}}^{a} \\
Y_{s_{2}}^{a}
\end{array}\right)+\left(\begin{array}{c}
v_{1} \\
\mathrm{M} \\
v_{m+s}+\lambda_{2}+\ldots+\lambda_{s_{1}}+v_{m+s+1}=1 \\
\lambda_{1}^{\prime}+\lambda_{2}^{\prime}+\ldots+\lambda_{s_{2}}^{\prime}+v_{m+s+2}=1 \\
\lambda_{1}, \lambda_{2}, \ldots, \lambda_{s_{1}} \geq 0 ; \lambda_{1}^{\prime}, \lambda_{2}^{\prime}, \ldots, \lambda_{s_{2}}^{\prime} \geq 0 ; v_{1}, \ldots, v_{m+s+2} \geq 0 \\
\mathrm{M} \\
0
\end{array}\right)
\end{array}\right.
\end{aligned}
$$

where $\lambda_{1}, \ldots, \lambda_{s_{1}}, \lambda_{1}^{\prime}, \ldots, \lambda_{s_{2}}^{\prime}$ are original unknowns and $v_{1}, \ldots, v_{m+s+2}$ are slacks.

It is easy to check that $\lambda_{1}, \ldots, \lambda_{s_{1}}=0 ; \lambda_{1}^{\prime}, \ldots, \lambda_{s_{2}}^{\prime}=0 ; v_{1}, \ldots, v_{m+s}=0 ; v_{m+s+1}=1$; $v_{m+s+2}=1$ is a feasible solution in model (A-4) so it is always feasible. Using it, we have the following theorem:

Theorem A-1: There exists no intersection between $\Phi$ and $\Pi$, if and only if the optimal value of objective function in model (A-4) is larger than zero.

Proof: If the optimal value is zero, then there is a feasible solution such that $v_{1}=0, \ldots, v_{m+s+2}=0$. Thus $\Phi$ and $\Pi$ has at least one intersection. If the minimal value is larger than zero, suppose that $\Phi$ and $\Pi$ have one intersection so that there exist feasible $\lambda_{1}, \ldots, \lambda_{s_{1}}, \lambda_{1}^{\prime}, \ldots, \lambda_{s_{2}}^{\prime}$ such that $v_{1}=0, \ldots, v_{m+s+2}=0$. Then it is clear that this is a feasible solution of model (A-5) and thus the minimal value should be zero, which is a contradiction. Q.E.D. 
Thus if the optimal value of objective function in model (A-4) is larger than zero, there exists no feasible solution in (A-3). Therefore, we can have a sufficient condition to ensure there is no intersection between efficient and anti-efficient frontiers. We can similarly handle the case where the BCC is assumed for the anti-efficient frontier. 